This item was submitted to Loughborough's Research Repository by the author.

Items in Figshare are protected by copyright, with all rights reserved, unless otherwise indicated.

\title{
Disturbance observer based control for nonlinear systems
}

PLEASE CITE THE PUBLISHED VERSION

PUBLISHER

(C) IEEE

LICENCE

CC BY-NC-ND 4.0

REPOSITORY RECORD

Chen, Wen-Hua. 2008. "Disturbance Observer Based Control for Nonlinear Systems". figshare. https://hdl.handle.net/2134/3760. 
This item was submitted to Loughborough's Institutional Repository (https://dspace.lboro.ac.uk/) by the author and is made available under the following Creative Commons Licence conditions.

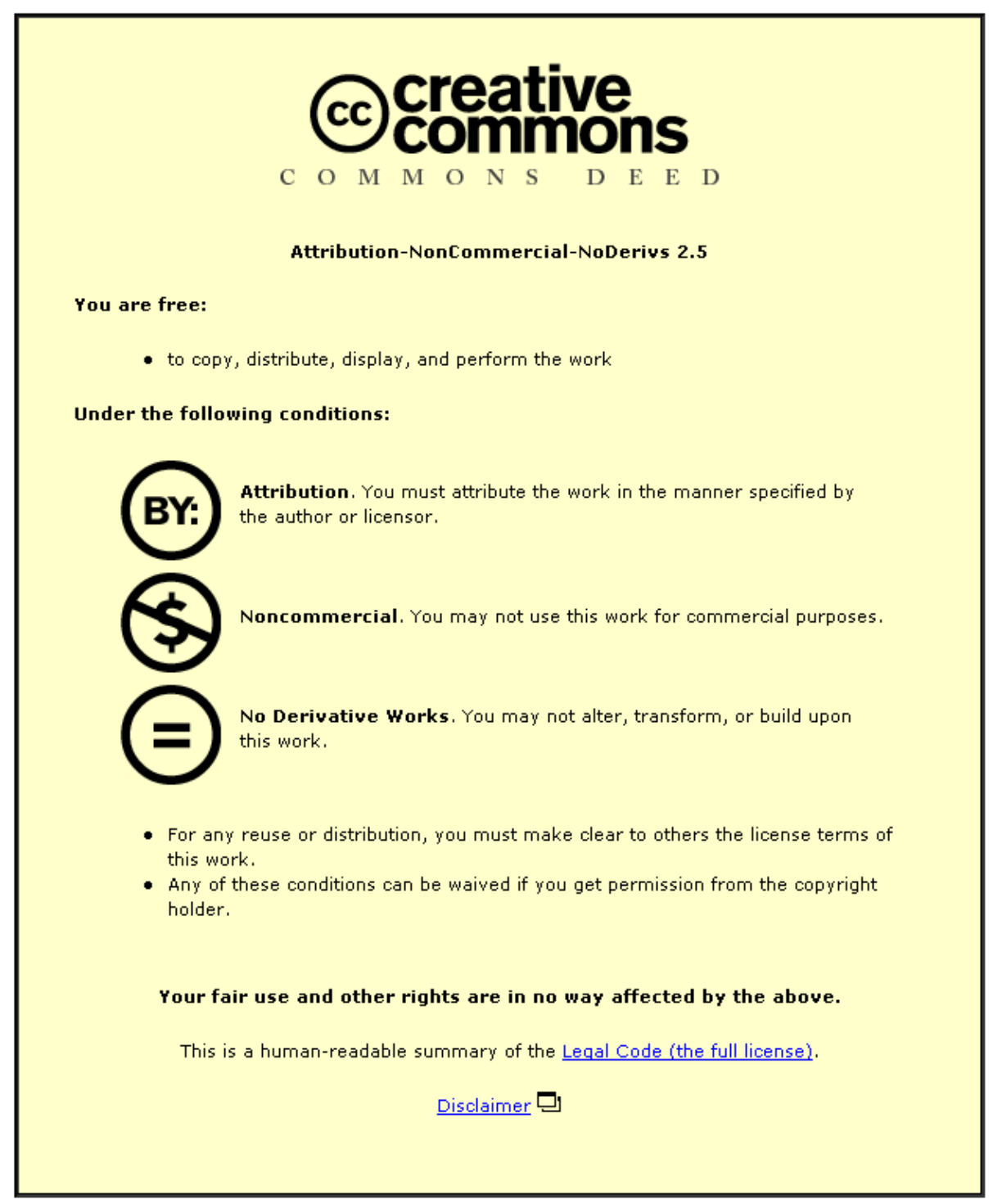

For the full text of this licence, please go to: http://creativecommons.org/licenses/by-nc-nd/2.5/ 


\section{Disturbance Observer Based Control for Nonlinear Systems}

\author{
Wen-Hua Chen
}

\begin{abstract}
This paper presents a general framework for nonlinear systems subject to disturbances using disturbance observer based control (DOBC) techniques. A two-stage design procedure to improve disturbance attenuation ability of current linear/nonlinear controllers is proposed where the disturbance observer design is separated from the controller design. To facilitate this concept, a nonlinear disturbance observer is developed for disturbances generated by an exogenous system, and global exponential stability is established under certain condition. Furthermore, semiglobal stability condition of the composite controller consisting of a nonlinear controller and the nonlinear disturbance observer is established. The developed method is illustrated by the application to control of a two-link robotic manipulator.
\end{abstract}

Index Terms-Composite controllers, control system design, disturbance attenuation, disturbance observers, nonlinear systems, stability.

\section{INTRODUCTION}

This paper proposes a disturbance observer based control (DOBC) approach for nonlinear systems under disturbances, namely, nonlinear DOBC or NDOBC. Within this framework, instead of considering the control problem for a nonlinear system under disturbances as a single one, it is divided into two subproblems, each with its own design objectives. The first subproblem is the same as the control problem for a nonlinear system without disturbances and its objective is to stablize the nonlinear plant and achieve performance specifications such as tracking or regulation. The second subproblem is to attenuate disturbances. A nonlinear disturbance observer is designed to deduce external disturbances and then to compensate for the influence of the disturbances using proper feedback. DOBC for linear systems has been developed and applied in engineering over two decades. Muller and Ackermann [1] and Nakao et al. [2] pioneered the development of DOBC for robot control independently. After that, DOBC has been applied in many mechatronic systems including disk drivers, machining centres, dc/ac motors, manipulators [3]-[5]. Most of the work in DOBC is engineering-oriented and lacks sound theoretic justification. When attempt is made to extend DOBC from linear systems to nonlinear systems, this leads to a composite controller consisting of a nonlinear controller and a nonlinear disturbance observer. Analysis and design of such a control system is challenging. Recent work has been concentrated on the development of nonlinear disturbance observers. To this end, Oh and Chung first improved a linear disturbance observer in robots using the information of nonlinear inertial coupling dynamics [6]. The application of this modified observer in redundant manipulators gives improved performance. A sliding mode based nonlinear disturbance observer was proposed and applied in motor control by [7]. Chen et al. [8] developed a nonlinear disturbance observer for unknown constant using Lyapunov theory and applied it to a two-link manipulator.

This paper attempts to establish a more general framework for NDOBC and establish rigorous basis for NDOBC development. First,

Manuscript received December 15, 2003; revised March 15, 2004. This work was supported by the U.K. Engineering and Physical Science Research Council under Grant GR/N31580.

The author is with the Department of Aeronautical and Automotive Engineering, Loughborough University, Loughborough, LE11 3TU, U.K. (e-mail: w.chen@lboro.ac.uk).

Digital Object Identifier 10.1109/TMECH.2004.839034 a general procedure for the design of DOBC for nonlinear systems is presented. Then, this procedure is applied to the control problem for a nonlinear system subject to disturbances generated by an exogenous system. This kind of disturbance widely exists in engineering including unknown load and harmonics, and has been investigated in several linear/nonlinear control methodologies, most notably, nonlinear output regulation theory [9].

There are three main contributions in this paper. The first one is that a general design procedure for DOBC of nonlinear systems is introduced. One of the main features of this two-stage design procedure is its flexibility and, in principle, it can be used to enhance the disturbance attenuation ability of any current nonlinear control method that cannot deal with disturbances. To establish rigorous theoretic results and facilitate this design concept, we then focus our attention on a class of nonlinear systems with disturbances generated by a general liner system. Many engineering problem can be formulated in this way; for example, nonlinear mechanical systems subject to unknown or variation of load and active noise/vibration control of nonlinear mechanical structures. The second contribution of this paper is to develop a nonlinear disturbance observer for this kind of disturbance and establish its global exponential stability. The traditional design methods of disturbance observers in linear DOBC are based on frequency domain techniques [10], which cannot be extended to nonlinear systems. A new methods for the nonlinear disturbance observer design is proposed in this paper using the algebraic structure of a nonlinear system. The third contribution is to establish semiglobal stability result for DOBC when the nonlinear disturbance observer is integrated with a nonlinear controller globally exponentially stabilizing the nonlinear system.

\section{NONLINEAR Disturbance OBSERVER BASED CONTROL (NDOBC)}

A nonlinear system is described by

$$
\left\{\begin{array}{l}
\dot{x}(t)=f(x(t))+g_{1}(x(t)) u+g_{2}(x(t)) d(t) \\
y(t)=h(x(t))
\end{array}\right.
$$

where $x \in R^{n}, u \in R$, and $d \in R$ are the state vector, input and external disturbance, respectively. It is assumed that $f(x), g_{1}(x), g_{2}(x)$ are smooth functions in terms of $x$.

A general design procedure for system (1) is proposed in the following NDOBC design procedure.

Step 1) Design a nonlinear controller for system (1) to achieve stability and other performance specifications under the assumption that the disturbance is measurable.

Stpe 2) Design a nonlinear disturbance observer to estimate the disturbance.

Step 3) Integrate the disturbance observer with the controller by replacing the disturbance in the control law with its estimation yielded by the disturbance observer.

The block diagram of the proposed NDOBC is shown in Fig. 1. As seen from this figure, the composite controller consists of two parts: a controller without or having poor disturbance attenuation ability and a disturbance observer.

Remark 1: The procedure proposed above consists of two stages. In the first stage, the controller is designed under the assumption that there is no disturbance or the disturbance is measurable. All the existing methods for designing a linear controller (for example, based linearised models) or a nonlinear controller can be used in this stage. In the second stage, a linear/nonlinear disturbance observer is designed and then integrated with the previous designed controller.

Remark 2: This procedure is quite general and flexible. Design of the controller is separated from design of the disturbance observer. In principle, it can be used to enhance the disturbance attenuation ability 


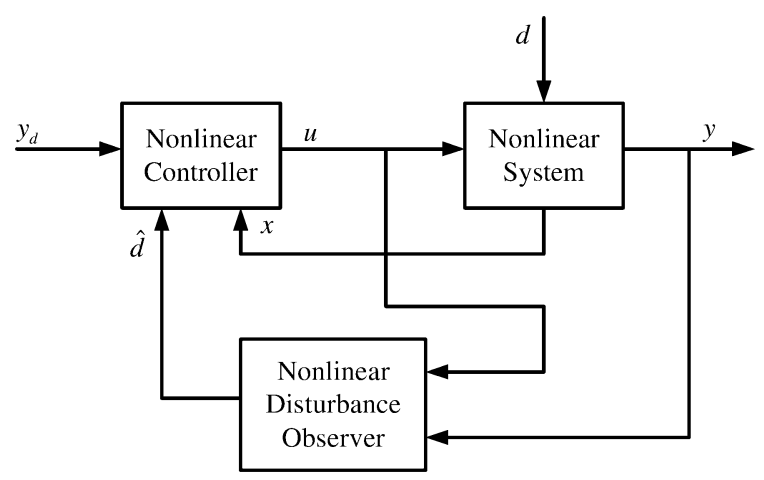

Fig. 1. Structure of nonlinear disturbance observer based control.

for any controller that cannot deal with disturbances or has poor disturbance attenuation ability.

Although the above approach is rather general, to establish theoretic results, we will concentrate on a class of nonlinear systems under disturbances. Furthermore, this paper will not discuss how to design a controller to stabilize nonlinear systems and achieve performance specifications for tracking or regulation in the absence of disturbances. It is assumed that a linear/nonlinear controller has been designed for this class of nonlinear systems using existing linear/nonlinear control techniques; for example, dynamic inversion control, feedback linearization, gain scheduling or sliding mode control. This paper will focus on Steps 2) and 3) in the design procedure, i.e., design of the nonlinear disturbance observer and integration of it with the controller. We also will investigate the property of the composite controller like stability.

\section{NONLINEAR DISTURBANCE OBSERVERS}

It is supposed that the disturbance is generated by an linear exogenous system

$$
\left\{\begin{array}{l}
\dot{\xi}=A \xi \\
d=C \xi
\end{array}\right.
$$

where $\xi \in R^{m}$ and $d \in R$. In general, the exogenous system (2) is assumed to be neutral stable, which implied that a persistent disturbance is imposed on the system (1).

To estimate the unknown disturbance $d$, a basic disturbance observer is suggested as

$$
\left\{\begin{array}{l}
\dot{\hat{\xi}}=A \hat{\xi}+l(x)\left(\dot{x}-f(x)-g_{1}(x) u-g_{2}(x) \hat{d}\right) \\
\hat{d}=C \hat{\xi}
\end{array}\right.
$$

where $l(x)$ is the nonlinear gain function of the observer.

However, the above disturbance observer cannot be implemented since the derivative of the state is required.

A new nonlinear disturbance observer is then proposed after modifying the above basic observer, given by (4) at the bottom of the page where $z \in R^{m}$ is the internal state variables of the observer and $p(x) \in R^{m}$ is a nonlinear function to be designed. The nonlinear observer gain $l(x)$ is then determined by

$$
l(x)=\frac{\partial p(x)}{\partial x} .
$$

Theorem 1: Consider system (1) under the disturbance generated by the exogenous system (2). The disturbance observer (4) can exponentially track the disturbance if the nonlinear gain function $l(x)$ is chosen such that

$$
\dot{e}(t)=\left(A-l(x) g_{2}(x) C\right) e(t)
$$

is globally exponentially stable regardless of $x$ where $e$ is the estimation error, defined as

$$
e=\xi-\hat{\xi}
$$

Proof: Combining (1) and (2), and (4)-(6) gives

$$
\begin{aligned}
\dot{e} & =\dot{\xi}-\dot{\hat{\xi}} \\
& =A \xi-\left(\dot{z}+\frac{\partial p}{\partial x} \dot{x}\right) \\
& =A e-l(x)\left(g_{2}(x) d-g_{2}(x) \hat{d}\right) \\
& =\left(A-l(x) g_{2}(x) C\right) e .
\end{aligned}
$$

This implies that $\hat{\xi}(t)$ approaches $\xi(t)$ exponentially if $l(x)$ is chosen such that (6) holds regardless of $x$. Hence, the result.

Q.E.D.

Suppose that the relative degree from the disturbance to the output, $r$, is uniformly well defined. This implies that $L_{g_{2}} L_{f}^{r-1} h(x) \neq 0$ for all $x$, where $L$ denotes Lie derivatives [11]. Without loss of generality, suppose that $L_{g_{2}} L_{f}^{r-1} h(x)>0$. This implies that $L_{g_{2}} L_{f}^{r-1} h(x)$ can be divided as

$$
L_{g_{2}} L_{f}^{r-1} h(x)=\alpha_{0}+\alpha_{1}(x)
$$

where $\alpha_{0}>0$ is a constant that can be chosen as the minimum of the function $L_{g_{2}} L_{f}^{r-1} h(x)$ over all $x$, and $\alpha_{1}(x)>0$ for all $x$. After the nonlinear variable $p(x)$ is chosen as

$$
p(x)=K L_{f}^{r-1} h(x)
$$

where $K=\left[k_{1}, \ldots, k_{m}\right]^{T}$ are gains to be determined, it follows from (5) that the observer gain function $l(x)$ is determined by

$$
l(x)=\frac{\partial p(x)}{\partial x}=K \frac{\partial L_{f}^{r-1} h(x)}{\partial x} .
$$

Then substitution of the above observer gain, together with (9), into the observer error dynamics (6) yields

$$
\begin{aligned}
\dot{e} & =\left(A-K \frac{\partial L_{f}^{r-1} h(x)}{\partial x} g_{2}(x) C\right) e \\
& =\left(A-K L_{g_{2}} L_{f}^{r-1} h(x) C\right) e \\
& =\left(A-K\left(\alpha_{0}+\alpha_{1}(x)\right) C\right) e .
\end{aligned}
$$

Theorem 2: The estimation yielded by the nonlinear disturbance observer (4) converges to the disturbance $d$ globally exponentially if there exists a gain $K$ such that the transfer function

$$
H(s)=C(s I-\bar{A})^{-1} K
$$

is asymptotically stable and strictly positive real where

$$
\bar{A}=\left(A-K \alpha_{0} C\right) .
$$

$$
\left\{\begin{array}{l}
\dot{z}=\left(A-l(x) g_{2}(x) C\right) z+A p(x)-l(x)\left(g_{2}(x) C p(x)+f(x)+g_{1}(x) u\right) \\
\hat{\xi}=z+p(x) \\
\hat{d}=C \hat{\xi}
\end{array}\right.
$$


Proof: According to strictly positive real Lemma, the transfer function (13) being stable and positive real implies that there exists a positive definite matrix $P$ such that

$$
\bar{A}^{T} P+P \bar{A}<0
$$

and

$$
P K=C^{T}
$$

Let an Lyapunov candidate for the observer error dynamics (6) be given by

$$
V_{O}(e)=e^{T} P e .
$$

Differentiation of the Lyapunov function with respect to time along the trajectory of the observer error dynamics (12) gives

$$
\begin{aligned}
\dot{V}_{O}(e) & =2 e^{T} P\left(A-K\left(\alpha_{0}+\alpha_{1}(x)\right) C\right) e \\
& =e^{T}\left(\bar{A}^{T} P+P \bar{A}\right) e-2 e^{T} P K C e \alpha_{1} \\
& <-\delta e^{T} e-2 e^{T} C^{T} C e \alpha_{1}(x)
\end{aligned}
$$

where $\delta$ is a small positive scalar depending on (15). The last inequality follows form condition (15) and (16). Since the relative degree from the disturbances to the output is uniformly well defined, it follows from (9) that $\alpha_{1}(x)>0$ regardless of $x$. Noting that $e^{T} C^{T} C e \geq 0$, (18) becomes

$$
\dot{V}_{O}(e)<-\delta e^{T} e
$$

for any $x$ and $e$, which implies that the estimation yielded by the disturbance observer approaches to the disturbance $d$ globally exponentially.

Q.E.D.

Theorem 2 states that the convergence of the disturbance observer regardless of $x$ can be guaranteed by determining the gain $K$ such that the transfer function (13) is asymptotical stable and strictly positive real. According to the proof, this can be performed by finding a suitable $P$ and $K$ such that conditions (15) and (16) are satisfied. Invoking (14), together with substitution of condition (16) into (15), it can be shown that conditions (15) and (16) are met if

$$
A^{T} P+P A-2 \alpha_{0} C^{T} C<0
$$

which is easy to calculate using linear matrix inequalities (LMIs) packages. After $P$ is determined, the gain can be calculated by

$$
K=P^{-1} C^{T}
$$

\section{Composite Controller}

After the nonlinear disturbance observer is designed as in (4), it is integrated with a separately designed controller. This section will investigate stability of the closed-loop system under the composite controller as shown in Fig. 1. The main result of this section is stated in Theorem 3.

Theorem 3: Consider nonlinear system (1) with well defined disturbance-to-output relative degree and under the disturbance (2). The closed-loop system under the nonlinear composite controller, namely, NDOBC, designed by the procedure in Section II, as shown in Fig. 1, is semiglobally exponentially stable in the sense that for an initial state $x$ and $\xi$ satisfying

$$
\|x(0)\| \leq R_{1} \quad\|\xi(0)\| \leq R_{2}
$$

where $R_{1}$ and $R_{2}$ are given scalars (could be arbitrarily large)

$$
\lim _{t \rightarrow \infty} x(t) \rightarrow 0
$$

and

$$
\lim _{t \rightarrow \infty} e(t) \rightarrow 0
$$

if the following conditions are satisfied:

1) when the disturbance $d$ is measurable, there exists a control law $u(x, d)$ such that the closed-loop system is globally exponentially stable for any disturbance;

2) the nonlinear disturbance observer (4) is designed as in Section III with the chosen design function $p(x)$ in (10) and there exists a gain function $K$ such the transfer function (13) is asymptotically stable and strictly positive real.

Proof: See the Appendix.

\section{EXAMPLE STUDY}

The proposed NDOBC approach is demonstrated by control of a two-link robotic manipulator, each link of which is directly driven by a dc motor [8]. The dynamic model of the manipulator including the first order dynamics of dc motors is given by

$$
J(\theta(t)) \ddot{\theta}(t)+G(\theta(t), \dot{\theta}(t))=u(t)+d(t)^{\prime}
$$

where $\theta \in R^{2}, \dot{\theta} \in R^{2}$ are displacement and velocity of the links. $u(t) \in R^{2}$ is the voltage applied on the motors and $d^{\prime}$ the equivalent torque disturbances on the motor input. In this simulation study, it is assumed that there is only reaction torque from the object/environment acting on the tip of the second link in the operation. Hence $d^{\prime}$ can be written as

$$
d^{\prime}(t)=c_{2} d(t)
$$

where

$$
c_{2}=\left[\begin{array}{l}
0 \\
1
\end{array}\right] \text {. }
$$

Following the design procedure in Section II, first it is assumed that there are no disturbances and the well-known computed torque control (CTC) method is applied to control of the two link robotic manipulator. The resulted control law is given by

$u^{*}(t)=-\left\{J(\theta)\left(K_{p}\left(\theta-\theta_{d}\right)+K_{v}\left(\dot{\theta}-\dot{\theta}_{d}\right)-\ddot{\theta}_{d}\right)-G(\theta, \dot{\theta})\right\}$

where $\theta_{d}$ defines reference trajectory of the manipulator, and $\dot{\theta}_{d}$ and $\ddot{\theta}_{d}$ are velocity and acceleration of the reference trajectory. The feedback gain matrices $K_{p}=\operatorname{diag}\left\{k_{p_{1}}, k_{p_{2}}\right\}$ and $K_{v}=\operatorname{diag}\left\{k_{v_{1}}, k_{v_{2}}\right\}$ are determined according to closed-loop performance requirements.

It is shown in Figs. 2 and 3 by the dashed lines (which are almost indistinguishable from solid lines) that in the absence of disturbance, satisfactory performance can be achieved by CTC with feedback gains

$$
k_{p_{i}}=7.5, \quad k_{v_{i}}=30 ; \text { for } i=1,2 \text {. }
$$

Suppose that there is a periodic disturbance torque acting on the end of the second link, given by

$$
d(t)=0.5 \sin (2 t+1) \mathrm{N} \cdot \mathrm{m} .
$$




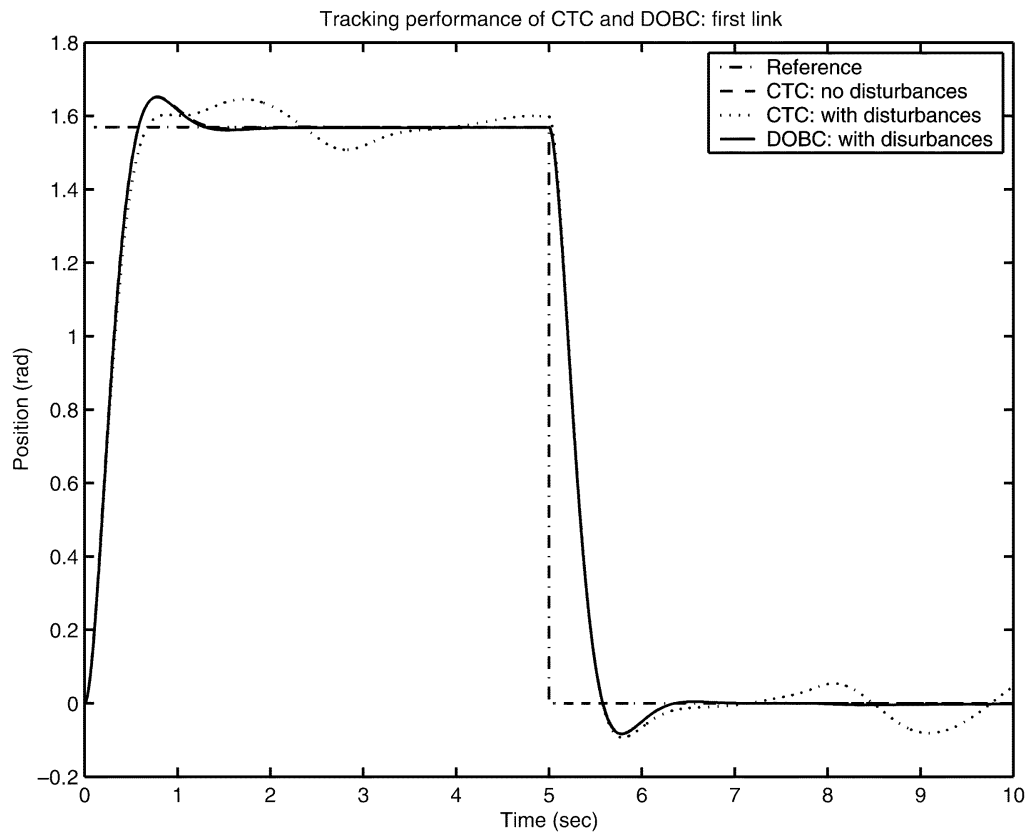

Fig. 2. Performance of disturbance observer enhanced computed torque controller: Link 1.

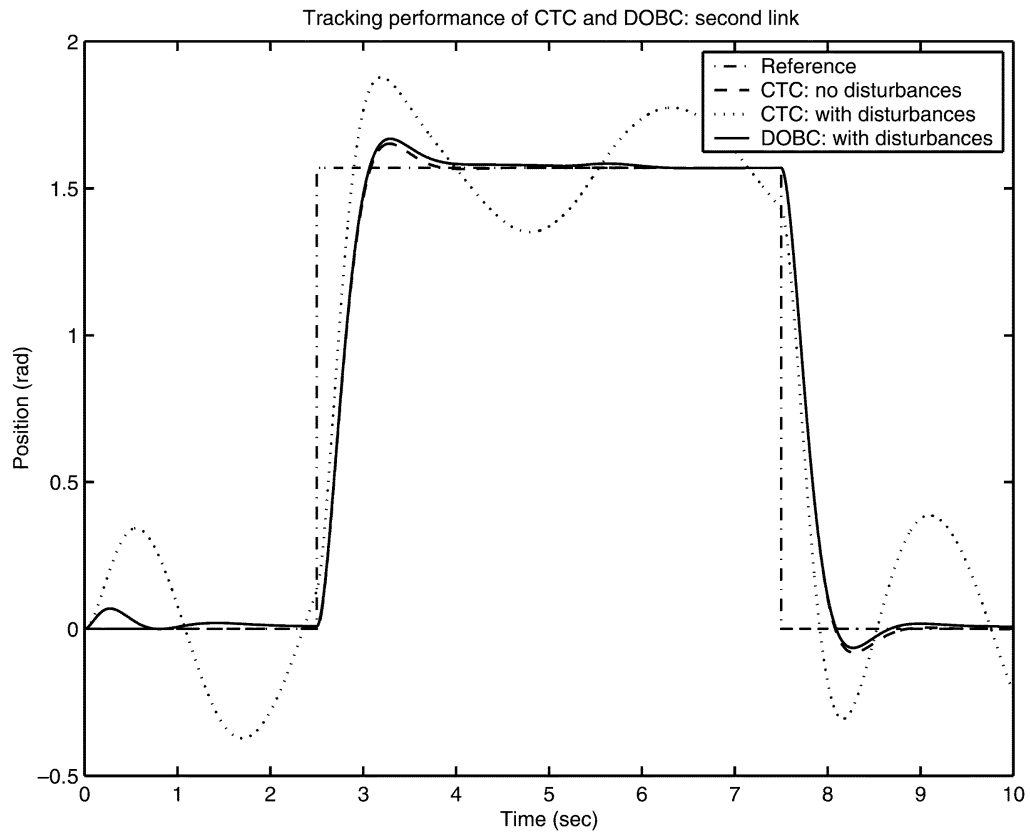

Fig. 3. Performance of disturbance observer enhanced computed torque controller: Link 2.

As is shown in Figs. 2 and 3 by the dotted lines, the performance of the above CTC is significantly degraded, in particular for the second link.

We now design a nonlinear disturbance observer to enhance the disturbance attenuation ability of the CTC by following the design procedure presented in Section III. The disturbance $d(t)$ can be generated by a neutral stable system described by (2) with

$$
A=\left[\begin{array}{cc}
0 & 2 \\
-2 & 0
\end{array}\right] \quad C=\left[\begin{array}{ll}
1 & 0
\end{array}\right]
$$

Following design procedure, the the nonlinear variable $p(x)$ can be chosen as

$$
p(x)=K L_{f} h(x)=K \dot{\theta}_{2}
$$

where $K=\left[\begin{array}{l}k_{1} \\ k_{2}\end{array}\right]$ is the gain matrix and is chosen as $k_{1}=k_{2}=10$ in the simulation.

The above designed disturbance observer is then integrated with the CTC to enhance its disturbance attenuation ability. The simulation results shown in Figs. 2 and 3 by the solid lines for Link 1 and 2, respectively, indicates that the performance of the CTC under the disturbance 
is significantly improved and the NDOBC achieves good disturbance attenuation ability. For NDOBC, there is tracking error caused by the disturbance in the second link at the beginning. However, after a short period, good tracking performance is achieved.

\section{CONCLUSION}

This paper proposes a general framework for design of controllers for nonlinear systems under disturbances using DOBC techniques. It is shown that this approach is quite flexible and can be integrated with current linear/nonlinear control methods that have poor disturbance attenuation ability. The effectiveness of the nonlinear DOBC procedure is illustrated by the control problem of a class of nonlinear systems subject to disturbances generated by a linear exogenous system. Theoretic results have been obtained for the DOBC approach to this problem including the global exponential stability of the nonlinear disturbance observer and semiglobal exponential stability of the composite controller.

\section{APPENDIX}

\section{A. Proof of Theorem 3}

In order to asymptotically stabilize the system for any disturbance, a part of the control effort, $u(x, d)$, shall linearly depend on the disturbances $d$. Thus, the control law can be divided into

$$
u(x, d)=\beta(x)+\gamma(x) d .
$$

Substituting (33) into system (1) obtains

$$
\dot{x}=f(x)+g_{1}(x) \beta(x)+g_{1}(x) \gamma(x) d+g_{2}(x) d
$$

which implies that in order to exponentially stabilize system (1) under arbitrary disturbance, there must exist $\gamma(x)$ such that

$$
g_{1}(x) \gamma(x)=-g_{2}(x)
$$

Under this condition, the closed-loop system (34) reduces to

$$
\dot{x}=f(x)+g_{1}(x) \beta(x)
$$

which is globally exponentially stable under an appropriately designed $\beta(x)$.

Since the disturbance $d$ is unavailable, it is estimated by the nonlinear disturbance observer (4). It follows from Theorem 2 that condition (2) in Theorem 3 implies that the nonlinear disturbance observer (4) with the design function (10) is globally exponentially stable. After replacing the disturbance $d$ by its estimate in the control law (33), according to condition (35), the closed-loop system (34) becomes

$$
\dot{x}=f(x)+g_{1}(x) \beta(x)+g_{2}(x)(d-\hat{d}) .
$$

Augmenting (37) with the observer dynamics (6) gives the closedloop system under the composite controller, described by

$$
\left\{\begin{array}{l}
\dot{x}=f(x)+g_{1}(x) \beta(x)+g_{2}(x) e_{1} \\
\dot{e}=\left(A-l(x) g_{2}(x) C\right) e
\end{array}\right.
$$

where $e_{1} \triangleq d-\hat{d}=C(\xi-\hat{\xi})=C e$. We now prove the semiglobal stability of the above composite system.

Since the system (36) is globally exponentially stable, it implies that there exists an Lyapunov function $V_{c}(x)$ such that its derivative along system (36) satisfies

$$
\dot{V}_{c}(x)=\frac{\partial V_{c}(x)}{\partial x}\left(f(x)+g_{1}(x) \beta(x)\right)<\delta_{1}\|x\|
$$

where $\delta_{1}$ is a small positive scalar. Choosing

$$
V(x, e)=V_{c}(x)+\mu V_{o}(e)=V_{c}(x)+\mu e^{T} P e
$$

as an Lyapunov candidate for system (38) where $\mu$ is a large positive scalar to be determined, one has

$$
\begin{aligned}
\dot{V}(x, e)=\frac{\partial V_{c}(x)}{\partial x}\left(f(x)+g_{1}(x) \beta(x)\right. & \left.+g_{2}(x) e_{1}\right) \\
& +2 \mu e^{T}\left(A-l(x) g_{2}(x) C\right) e .
\end{aligned}
$$

When the transfer function (13) is asymptotically stable and strictly positive real, substitution of (19) and (39) into (41) yields

$$
\begin{aligned}
\dot{V}(x, e)= & \frac{\partial V_{c}(x)}{\partial x}\left(f(x)+g_{1}(x) \beta(x)\right)+\frac{\partial V_{c}(x)}{\partial x} g_{2}(x) e_{1} \\
& -\mu \delta e^{T} e \\
< & -\delta_{1}\|x\|+\frac{\partial V_{c}(x)}{\partial x} g_{2}(x) e_{1}-\mu \delta e^{T} e .
\end{aligned}
$$

In the same fashion as in [11], one can conclude that all the state and observer error starting from a possible arbitrarily large set converges to the origin as $t \rightarrow \infty$. Hence, the results.

QED

\section{REFERENCES}

[1] P. C. Muller and J. Ackermann, "Nichtlineare regelung von elastischen robotern," in VDI-Berichte 598, Steuerung und Regelung von Roboter. Berlin, Germany: Springer-Verlag, 1986, pp. 321-333.

[2] M. Nakao, K. Ohnishi, and K. Miyachi, "Robust decentralized joint control based on interference estimation," in Proc. IEEE Int. Conf. Robotics and Automation, Raleigh, NC, 1987, pp. 326-333.

[3] C. J. Kemf and S. Kobayashi, "Disturbance observer and feedforward design for a high-speed direct-drive positioning table," IEEE Trans. Contr. Syst. Technol., vol. 7, pp. 513-526, Sept. 1999.

[4] Y. H. Huang and W. Messner, "A novel disturbance observer design for magnetic hard drive servo system with rotary actuator," IEEE Trans. Magn., vol. 4, pp. 1892-1894, July 1998.

[5] J. Ishikawa and M. Tomizuka, "Pivot friction compensation using an accelerometer and a disturbance observer for hard disk," IEEE/ASME Trans. Mechatron., vol. 3, pp. 194-201, Sept. 1998.

[6] Y. Oh and W. K. Chung, "Disturbance-observer-based motion control of redundant manipulators using inertially decoupled dynamics," IEEE/ASME Trans. Mechatron., vol. 4, pp. 133-145, Dec. 1999.

[7] X. Chen, S. Komada, and T. Fukuda, "Design of a nonlinear disturbance observer," IEEE Trans. Ind. Electron., vol. 47, pp. 429-436, Apr. 2000.

[8] W.-H. Chen, D. J. Ballance, P. J. Gawthrop, and J. O’Reilly, "A nonlinear disturbance observer for two-link robotic manipulators," IEEE Trans. Ind. Electron., vol. 47, pp. 932-938, Aug. 2000.

[9] A. Isidori and C. J. Byrnes, "Output regulation of nonlinear systems," IEEE Trans. Automat. Contr., vol. 35, pp. 131-140, Feb. 1990.

[10] T. Mita, M. Hirata, K. Murata, and H. Zhang, " $H_{\infty}$ control versus disturbance observer based control," IEEE Trans. Ind. Electron., vol. 45, pp. 488-495, June 1998.

[11] A. Isidori, Nonlinear Control Systems: An Introduction, 3rd ed. New York: Springer-Verlag, 1995. 